\title{
Review Article \\ Inositol Treatment and ART Outcomes in Women with PCOS
}

\author{
Deepika Garg' and Reshef Tal' \\ ${ }^{1}$ Department of Obstetrics and Gynecology, Maimonides Medical Center, Brooklyn, NY, USA \\ ${ }^{2}$ Division of Reproductive Endocrinology \& Infertility, Department of Obstetrics, Gynecology and Reproductive Sciences, \\ Yale University School of Medicine, New Haven, CT, USA \\ Correspondence should be addressed to Reshef Tal; reshef.tal@yale.edu
}

Received 19 June 2016; Accepted 30 August 2016

Academic Editor: Nikos Prapas

Copyright (C) 2016 D. Garg and R. Tal. This is an open access article distributed under the Creative Commons Attribution License, which permits unrestricted use, distribution, and reproduction in any medium, provided the original work is properly cited.

\begin{abstract}
Polycystic ovary syndrome (PCOS) affects $5-10 \%$ of women in reproductive age and is characterized by oligo/amenorrhea, androgen excess, insulin resistance, and typical polycystic ovarian morphology. It is the most common cause of infertility secondary to ovulatory dysfunction. The underlying etiology is still unknown but is believed to be multifactorial. Insulin-sensitizing compounds such as inositol, a B-complex vitamin, and its stereoisomers (myo-inositol and D-chiro-inositol) have been studied as an effective treatment of PCOS. Administration of inositol in PCOS has been shown to improve not only the metabolic and hormonal parameters but also ovarian function and the response to assisted-reproductive technology (ART). Accumulating evidence suggests that it is also capable of improving folliculogenesis and embryo quality and increasing the mature oocyte yield following ovarian stimulation for ART in women with PCOS. In the current review, we collate the evidence and summarize our current knowledge on ovarian stimulation and ART outcomes following inositol treatment in women with PCOS undergoing in vitro fertilization (IVF) and/or intracytoplasmic sperm injection (ICSI).
\end{abstract}

\section{Introduction}

Polycystic ovarian syndrome (PCOS) is the most common endocrine disorder of women in reproductive age with a prevalence ranging from 5 to $10 \%$ [1]. According to the 2003 Rotterdam consensus workshop, it is defined by 2 out of the following 3 criteria: hyperandrogenism, oligo/anovulation, and polycystic ovarian morphology [2]. There is an increased risk of hypertension, cardiovascular disease, impaired glucose tolerance, type II diabetes, and dyslipidemia in women suffering from PCOS. Despite many decades of extensive research, the exact etiology of this disorder remains largely unknown, although it is recognized that ovarian hyperthecosis, increased androgens, insulin resistance, and genetic and environmental factors all play a role in the pathophysiology of this syndrome [3]. It is widely known that insulin resistance (IR) plays an important role in the pathogenesis of PCOS in a large subset of patients [4]. Insulin stimulates ovarian theca cells to produce and secrete androgens both directly and indirectly. Elevated glucose levels in turn inhibit the hepatic synthesis of sex hormone-binding globulin, leading to increased concentration of circulating free androgens [5].
Insulin resistance is a common feature in both overweight and lean women with PCOS independent of their body mass index (BMI) [6]. Approximately $80 \%$ of obese women with PCOS and $30-40 \%$ of lean women with PCOS have hyperinsulinemia due to IR [7]. As the relationships between IR and PCOS manifestations were established, several insulinsensitizing compounds such as metformin and thiazolidinediones were demonstrated as effective adjuncts in treating PCOS women $[8,9]$. In addition, over the last decade the use of myo-inositol, a natural insulin sensitizer, has been investigated in many studies of PCOS women. The role of myo-inositol in women with PCOS can be attributed to deficiency of metabolites of myo-inositol which are mediators of insulin action [10]. In addition to the beneficial effects of myo-inositol on insulin resistance and other metabolic aspects, it has been shown to improve oocyte maturation, follicular milieu, and outcomes of ART in women with PCOS $[11,12]$. In this article, we review the effects of myo-inositol on ovulatory dysfunction in women with PCOS with a particular focus on the accumulating evidence associating myo-inositol with ART outcome in these women. Better understanding of 
the role of myo-inositol in the reproductive function in PCOS may improve ART outcomes in women with PCOS.

\section{Methods}

A comprehensive literature search was performed using the electronic databases PubMed, Medline, and Google Scholar for relevant articles in the English language until May 2016. The following combinations of search terms were used: "polycystic ovarian syndrome", "PCOS", "inositol", "ovulatory dysfunction", "hyperandrogenism”, "insulin resistance”, "ovulation induction", "in vitro fertilization", "IVF", "Assisted reproductive technology", "ART", "metformin", and "treatment outcome". The abstracts identified were reviewed and evaluated by both authors. Any disagreement between the two researchers was resolved with discussion. The reference lists of the selected papers were manually searched in order to identify additional potentially relevant studies.

2.1. Inositols: Biochemistry and Sources. Inositols and their derivatives are sugar alcohols which belong to the vitamin B family, representing nine cyclohexane-1,2,3,4,5,6-hexol stereoisomers. They are chemically stable molecules which can be ingested from the diet [13]. Dietary products containing inositol include fruits, mainly cantaloupe and oranges, high bran content cereals, nuts, and beans. Fresh fruits and vegetable contain more inositol when compared with the frozen, salted, or canned products. The average daily intake of myo-inositol from the diet may vary between 225 and $1500 \mathrm{mg} /$ day per $1800 \mathrm{kcal}$ diet [14]. Inositols are not considered an essential nutrient since they can be formed endogenously from glucose [15]. Myo-inositol (MYO) and D-chiro-inositol (DCI) are the two out of nine existing stereoisomers of inositol that are formed after epimerization of the hydroxyl groups of inositol [12, 16-18]. Myo-inositol is the most abundant form of inositol in both nature and mammalian cells, comprising up to $99 \%$ of the inositol amount. The remaining $1 \%$ is represented by the other stereoisomer DCI. Endogenously, the synthesis of myoinositol occurs from glucose-6-phosphate in the two-step process which involves conversion of glucose-6-phosphate to myo-inositol 1-phosphate by inositol-3-phosphate synthase enzyme, which is then dephosphorylated to form free myo-inositol by the enzyme inositol monophosphatase [19]. Myo-inositol is converted into DCI by an NAD/NADH epimerase. The activity of this insulin-dependent enzyme strongly influences the intracellular ratio between these two molecules in adipose, hepatic, or muscle cells [20]. Both of these molecules have been used as insulin-sensitizing agents in women with PCOS [21, 22]. Inositol is present in phospholipids, can stimulate lecithin production, serves as an important component of structural lipids, and also controls the fat and lipid metabolism.

2.2. Inositol and Reproductive Function. Inositols and their phosphates function as secondary messenger molecules for various cellular signaling pathways including insulin signal transduction, calcium trafficking, lipid metabolism, cytoskeletal protein assembly, cell growth and differentiation, modulation of serotoninergic pathways, oocyte maturation, and fertility [29]. Uptake of free inositol by tissues occurs by a membrane dependent sodium-inositol cotransporter [30]. However, in comparison to DCI, MYO has 10 times greater affinity for this cotransporter [31]. There is substantial evidence linking inositols to reproductive outcomes. Inositol has been demonstrated to play an important role in oocyte maturation and fertilization via its regulation of calcium signaling pathways $[32,33]$. Inositol 1,4,5-trisphosphate receptor (IP3R) plays a key role in triggering the $\mathrm{Ca}^{2+}$ release during oocyte maturation and fertilization [34, 35]. Myoinositol levels in blood and follicular fluid have been shown to positively correlate with oocyte quality and pregnancy outcome in humans $[32,36]$. Moreover, supplementation of MYO has been suggested to promote meiotic progression of oocytes, producing better quality oocytes and embryos in mice $[37,38]$. In humans, administration of MYO to women prior to hormonal stimulation in IVF cycles has been shown to increase oocyte and embryo quality and reduce the dose of FSH and the days required for stimulation [11, 39]. Furthermore, studies on PCOS women have shown that MYO can improve menstrual regularity, insulin resistance, and oocyte quality and maturation $[23,40,41]$.

2.3. Inositol, Insulin Resistance, and Ovarian Function in PCOS. It is now recognized that insulin resistance has a critical role in the pathogenesis of PCOS. Hyperinsulinemia secondary to insulin resistance is common in a high proportion of PCOS patients and is associated with metabolic morbidities as well as reproductive dysfunction [42]. Insulin resistance is thought to contribute to hyperandrogenism in PCOS by insulin stimulating ovarian theca cells to produce and secrete androgens and by elevated glucose levels inhibiting the hepatic synthesis of sex hormone-binding globulin, leading to increased concentration of circulating free androgens [5]. While the exact cause of insulin resistance in PCOS is unknown, it has been postulated that a defect in the postreceptor transport of glucose and selective resistance to metabolic actions of insulin may be responsible for hyperinsulinemia in these patients $[43,44]$. The well-known association between hyperinsulinemia, hyperandrogenism, and ovulatory dysfunction in PCOS formed a basis for treatment with insulin-sensitizing agents such as myo-inositol, metformin, and thiazolidinediones, which have proven effective in improving insulin resistance as well as ovarian functions in these women [45].

Inositol and its stereoisomers are classified as insulin sensitizers and act as a second messenger of insulin signaling pathways [46]. Indeed, the actions of insulin are mediated by two distinct inositol phosphoglycan (IPG) mediators, incorporating either MYO or DCI, which are released by the hydrolysis of glycosyl-phosphatidyl-inositol lipids on the outer side of the cell membrane. IPG in turn activates the key enzymes controlling the oxidative and nonoxidative glucose metabolism, affecting the intracellular metabolic processes. Both MYO- and DCI-containing IPGs decrease insulin resistance [47].

Women with PCOS have reduced serum level and increased urinary loss of DCI [48, 49]. It was demonstrated that DCI urinary clearance was inversely correlated with 
insulin sensitivity in PCOS women and was a strong and independent predictor of insulin resistance in these women $[48,49]$. Following these observations, it has been established that PCOS women have a dysregulation of inositol metabolism [50], providing a mechanistic link between inositol deficiency and insulin resistance in PCOS. Since IPG signaling pathways are involved in insulin-mediated thecal androgen biosynthesis, defective conversion of MYO to DCI in PCOS patients may also contribute to hyperandrogenism [51]. Indeed, administration of DCI at low doses has been shown to decrease insulin resistance and serum androgens and improve ovulatory frequency in PCOS women [16, 52, 53]. However, when administered at higher doses DCI appears to exert negative effects on the ovaries [54]. In fact, subsequent clinical trials performed with DCI doses of $2.4 \mathrm{~g} /$ day were unable to confirm previous positive results on PCOS women, suggesting that DCI may paradoxically worsen the ovarian response in these patients despite normalization of the IR parameters [53].

As mentioned earlier, MYO is converted to DCI via an insulin-induced epimerase enzyme in different tissues based on their requirement for each of these molecules [55, 56]. DCI conversion is reduced in muscle tissue of subjects suffering from insulin resistance due to decreased epimerase activity $[20,57,58]$. These studies included muscle and liver which can develop insulin insensitivity. However, in contrast to these tissues, normal ovaries and those of ovulatory PCOS remain insulin sensitive $[59,60]$. Moreover, one study demonstrated higher $\mathrm{M} / \mathrm{C}$ epimerase specific activity in theca cells of PCOS compared to control women and 4-fold higher MYO : DCI ratio in control than in PCOS [61]. Thus, hyperinsulinemia may alter the MYO : DCI ratio and paradoxically increase DCI concentration within the ovary. Decrease in MYO : DCI ratio has also been recently reported in follicular fluid of PCOS women [62]. These observations support the notion that any further increase in DCI is detrimental to ovarian function, explaining the lack of clinical benefit when using DCI in PCOS women and highlighting the importance of maintaining proper $\mathrm{MYO}$ : DCI ratio when administering inositols to PCOS women.

In contrast, the benefit of MYO supplementation in PCOS has been demonstrated by several studies. Administration of MYO combined with folic acid $2 \mathrm{~g}$ twice a day for 6 months in PCOS patients showed maintenance of normal ovulatory activity in $72 \%$, with singleton pregnancy rate of $40 \%$ during the 6-month observation period [23]. In a recent study by Kamenov et al., 50 anovulatory PCOS patients with insulin resistance were given MYO for three spontaneous cycles and ovulation and pregnancy were achieved in $61.7 \%$ and $37.9 \%$ of women, respectively. In the women who remained anovulatory, MYO was used in combination with clomiphene citrate for three cycles resulting in ovulation and pregnancy rates of $72.2 \%$ and $42.6 \%$, respectively. These patients also had reduction in BMI and HOMA index, suggesting the role of $\mathrm{MYO}$-induced amelioration of insulin resistance, in mediating the improvement in ovarian function in women with PCOS [63]. While the above studies lacked a control group, similar beneficial effects on ovarian function were also noted by Artini et al. in their randomized controlled trial of 50 overweight PCOS patients which were divided into two groups; group A was given MYO $2 \mathrm{~g}$ plus folic acid $200 \mathrm{mg}$ daily for 12 weeks and group B was given folic acid $200 \mathrm{mg}$ daily. They found significant improvement in hormonal parameters and restoration of menstrual cyclicity in all amenorrheic and oligomenorrheic patients in group $\mathrm{A}$ while no changes were noted in group $\mathrm{B}$, suggesting the role of MYO in improving the reproductive axis in PCOS patients [64]. Moreover, administration of both MYO plus DCI has been shown to be effective in achieving better clinical results in PCOS patients in a combination replicating the plasma physiological ratio $(40: 1)$ by working at systemic and ovary level $[62,65,66]$. The mechanism of the beneficial effects of MYO on ovarian function in the polycystic ovaries could be due to increase in glucose uptake and facilitating $\mathrm{FSH}$ signaling that likely improve oocyte quality and better IVF outcome [67].

Another important consideration when evaluating the effects of inositols in PCOS women is the interaction with obesity. Baillrgeon et al. showed that obese PCOS women have diminished release of DCI-IPG in response to insulin elevation compared to normal weight women [50]. Since this study did not include normal weight PCOS women, it is difficult to ascertain whether the observed abnormal DCI-IPG response was related to PCOS, obesity, or a combination of both. However, the investigators noted that reduced insulinstimulated DCI-IPG response was significantly associated with obesity (BMI, $r=-0.56, P=0.025$ ), suggesting that obesity plays a role in abnormal inositol metabolism independently of PCOS. In a randomized trial of inositol treatment in PCOS women, Gerli et al. have shown that metabolic risk factor benefits of inositol treatment were not observed in the morbidly obese subgroup of patients, noting an inverse relationship between BMI and treatment efficacy [12]. Recently, Ferrari et al. studied the effects of MYO/DCI supplementation on the maternal metabolic profile in mouse pregnancies complicated by obesity or metabolic syndrome [68]. For their metabolic syndrome model, they used their previously characterized female heterozygous +/- mice lacking endothelial nitric oxide synthase (eNOS), which after feeding with a high-fat diet for 4 weeks develop metaboliclike syndrome phenotype including obesity, glucose intolerance, elevated systolic blood pressure, low high-density lipoprotein, and high insulin. For obesity model, they used wild-type C57BL/6J female mice fed a high-fat diet from weaning for 4 weeks. The pregnant mice were randomized to receive either $\mathrm{MYO} / \mathrm{DCI}(7.2 / 0.18 \mathrm{mg} / \mathrm{mL}$, resp.) or water as placebo in control group. Pregnant mice with metaboliclike syndrome showed lower serum glucose levels and leptin levels following MYO/DCI treatment as compared to placebo group. In contrast, pregnant mice with obesity alone did not demonstrate improvement in any of the metabolic parameters as compared to placebo group [68]. It was speculated by the study's investigators that MYO/DCI treatment improves glucose tolerance in metabolic-like syndrome pregnant mice but not in the obese mice, possibly involving its specific effects on the nitric oxide pathway. While the above studies suggest that the beneficial effects of inositols may be reduced in obese population, the majority of studies evaluating the effects of inositol treatment on metabolic as well as reproductive function in PCOS women have not specifically addressed the 
potential interaction of treatment response with BMI. Future studies are needed to better characterize inositol effects as a function BMI and investigate the potential mechanism/s underlying this differential response.

2.4. Inositol and ART Outcomes in PCOS. Inositol plays an important role in the follicular microenvironment and affects oocyte maturation and embryo development [24]. Elevated concentration of MYO in the follicular fluid appears to exert a positive effect on follicular maturity and is a marker of good quality oocytes in women with or without PCOS $[25,69]$. Recent studies have evaluated the role of inositol in ART outcome in women with PCOS. The data from these studies support the notion that inositol has a beneficial effect on ovarian stimulation and ART outcomes in PCOS patients.

Papaleo et al. investigated the effect of MYO supplementation of $2 \mathrm{~g}$ twice a day on ART outcomes in sixty patients with PCOS undergoing ovarian stimulation for intracytoplasmic sperm injection (ICSI) cycles. They found significant reduction in the total number of days of stimulation (11.4 \pm 0.9 versus $12.4 \pm 1.4, P=0.01)$, significantly lower peak E2 levels at hCG administration (2,232 \pm 510 versus $2,713 \pm$ $595 \mathrm{pg} / \mathrm{mL}, P=0.02$ ), and reduction in degenerated oocytes $(1.0 \pm 0.9$ versus $1.6 \pm 1.0, P=0.01)$ without compromising oocyte yield in the myo-inositol group in comparison to folic acid alone group [11]. However, no differences were found in fertilization rate, embryo quality, or clinical pregnancy rates between the two groups. The authors also suggested that MYO supplementation may decrease the risk of ovarian hyperstimulation syndrome in PCOS patients [11].

Similar findings were reported by Ciotta et al. in a randomized study in which they evaluated the effects of myoinositol on oocyte and embryo quality in 34 PCOS patients undergoing IVF/ICSI. Patients in this study were divided into two groups: group A was given myo-inositol $(2 \mathrm{~g})$ and folic acid $(200 \mu \mathrm{g}) 2$ times a day for 3 months, while group B received only folic acid $(200 \mu \mathrm{g})$. Their results showed lower peak E2 levels at hCG administration, less cycle cancellation, higher number of oocytes retrieved, significantly lower number of immature oocytes, and better quality of embryos with higher number of transferred embryos in group A in comparison to group B [41]. Another recent randomized clinical trial by Unfer et al. aimed at comparing the effects of MYO to DCI on the oocyte and embryo quality in euglycemic patients with PCOS undergoing ovarian stimulation for ICSI. Out of eighty-four women with PCOS in their study, forty-three were given MYO $2 \mathrm{~g}$ twice a day and forty-one women were given DCI $0.6 \mathrm{~g}$ twice a day. The results showed significantly increased number of mature oocytes, good quality embryos, and total pregnancies in MYO-treated group in comparison to DCI treated group [70]. Similar negative effects of DCI were also noted in a study by Isabella and Raffone who investigated the role of DCI in 54 women diagnosed with PCOS undergoing ICSI. After excluding patients with insulin resistance and/or hyperglycemia, they were divided into 5 groups (10-12 patients/group) with a placebo group and 4 other groups receiving 300, 600, 1200, or $2400 \mathrm{mg}$ DCI daily for 8 weeks. They found significantly increased number of immature oocytes in the three groups that received the higher doses of DCI $(P<0.04)$, with significant reduction in grade I embryos $(P=0.004)$ in DCI supplementation group suggesting the negative effect of DCI on oocyte and embryo quality and worsening ovarian response with increasing DCI dosage in PCOS patients [54]. These data are consistent with the DCI paradox hypothesis, which suggests that in PCOS patients there is depletion of MYO due to accelerated epimerization from MYO to DCI and thus further increase in DCI may be accountable for poor folliculogenesis and oocyte response in these patients $[26,71]$. Similar paradoxical findings were noted in PCOS patients undergoing IVF and treated with metformin for 4-8 weeks where metformin reduced the number of oocytes retrieved [27]. The authors suggested that the mechanism behind their results may be increased DCI release in response to metformin [27].

Colazingari et al. studied the role of combined $\mathrm{MYO}$ and DCI in comparison to DCI alone in PCOS patients undergoing IVF. They included PCOS patients with BMI less than 28 and FSH less than $10 \mathrm{IU} / \mathrm{L}$ undergoing IVFET and treated them with MYO combined with DCI in a physiological ratio (1.1 g myo-inositol plus $27.6 \mathrm{mg}$ of Dchiro-inositol) or with DCI alone $(500 \mathrm{mg})$ for 12 weeks. They found reduced number of degenerated oocytes $(1.04 \pm 1.15$ versus $1.82 \pm 1.55)$, better fertilization rate $(0.75 \pm 0$ versus $0.58 \pm 0.29)(P<0.05)$, number of transferred embryos $(2.22$ \pm 0.74 versus $1.67 \pm 0.85, P<0.05)$, and improved embryo quality $(0.96 \pm 0.83$ versus $0.7 \pm 0.73, P<0.05)$ in MYO-DCI treated group in comparison to DCI only treated group [28].

In the largest study to date evaluating the effects of MYO supplementation in PCOS women undergoing IVF, Pacchiarotti et al. randomized 526 PCOS patients into three groups: control (folic acid: $400 \mathrm{mcg}, n=195$ ), group A (myoinositol: $4000 \mathrm{mg}$, folic acid: $400 \mathrm{mcg}$, and melatonin: $3 \mathrm{mg}$ daily, $n=165$ ), and group B (myo-inositol: $4000 \mathrm{mg}$ and folic acid: $400 \mathrm{mcg}$ daily, $n=166$ ). All patients received their treatment from first day of the menstrual cycle until 14 days after embryo transfer. Patients in group A required decreased dose of gonadotropins (group A $2058 \pm 233$ versus group B $3113 \pm 345$ versus control group $3657 \pm 633, P<0.001$ ) and had enhanced quality of oocytes (group A: $48.2 \%$ versus group B 35.0\% versus control group 38.2\%) and embryos (45.7\% in group A versus $30.4 \%$ in group B and $25.6 \%$ in the control group), suggesting the synergistic effect of MYO and melatonin on improving oocyte and embryo quality [72].

In another study by Rago et al. the combined effect of MYO and $\alpha$-lipoic acid was studied in PCOS patients with normal BMI who had received $\mathrm{MYO}$ alone and undergone ICSI previously. They reenrolled 36 PCOS patients who did not achieve pregnancy and 1 patient who had spontaneous abortion and supplemented them with MYO $(2 \mathrm{~g})$ and $\alpha$ lipoic acid $(800 \mathrm{mg})$ per day for 3 months. In MYO and $\alpha$-lipoic acid group, significant reduction was noted in immature oocytes $(0.2 \pm 0.4$ versus $1.0 \pm 1.5 ; P<0.001)$, with improvement in mature oocytes $(0.87 \pm 0.9 \%$ versus $0.81 \pm 3.9 \%, P<0.05)$ and increase in grade 1 embryos (75.7\% versus $57.7 \% ; P<0.05$ ) and higher number of pregnancies achieved (52\% versus 33.3\%; $P<0.01$ ) in comparison to MYO alone group [73]. While this study is limited by lack of a control group, the results suggest that $\alpha$-lipoic acid may enhance the beneficial effects of MYO in PCOS women. Table 1 summarizes the results of the clinical 


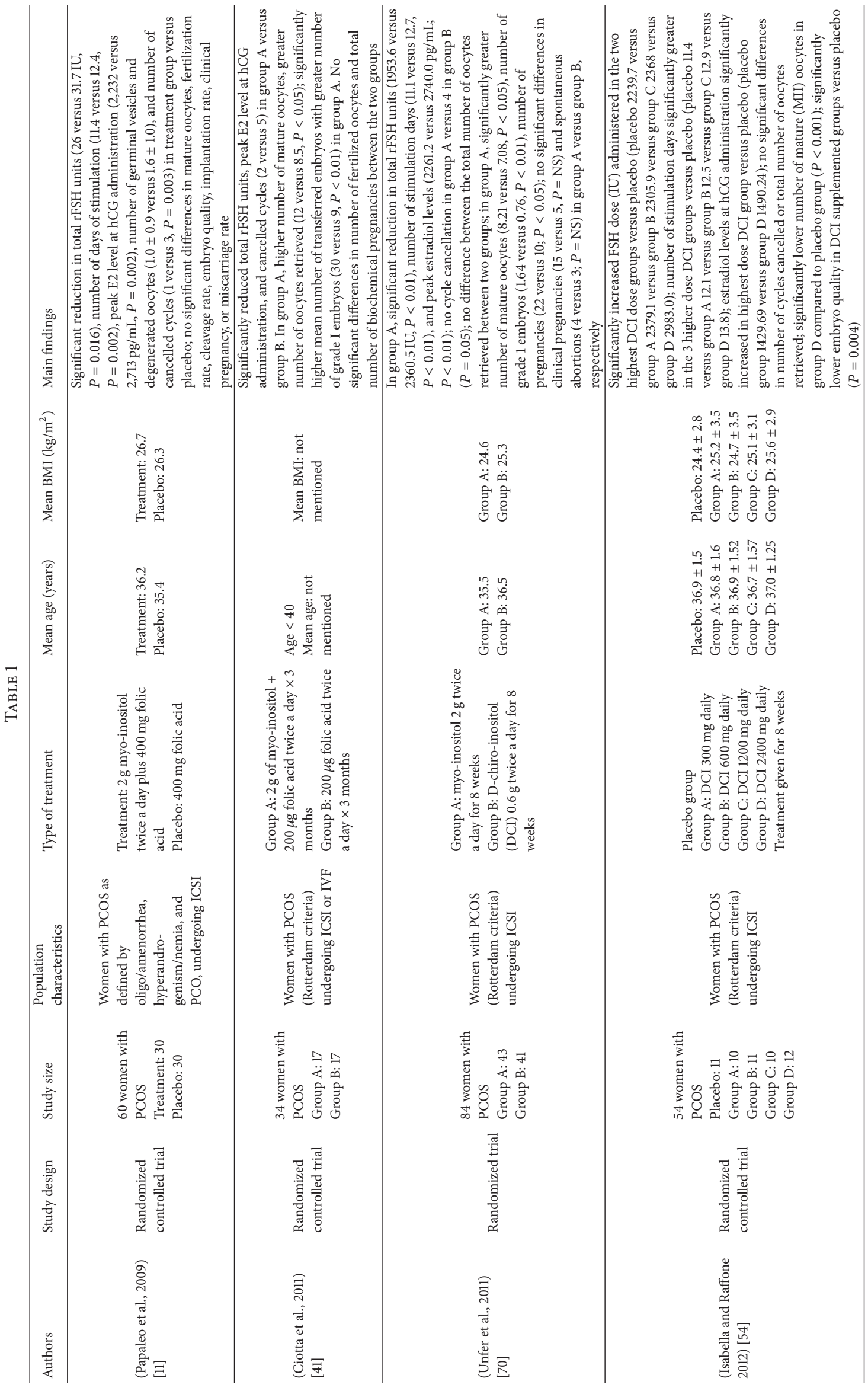




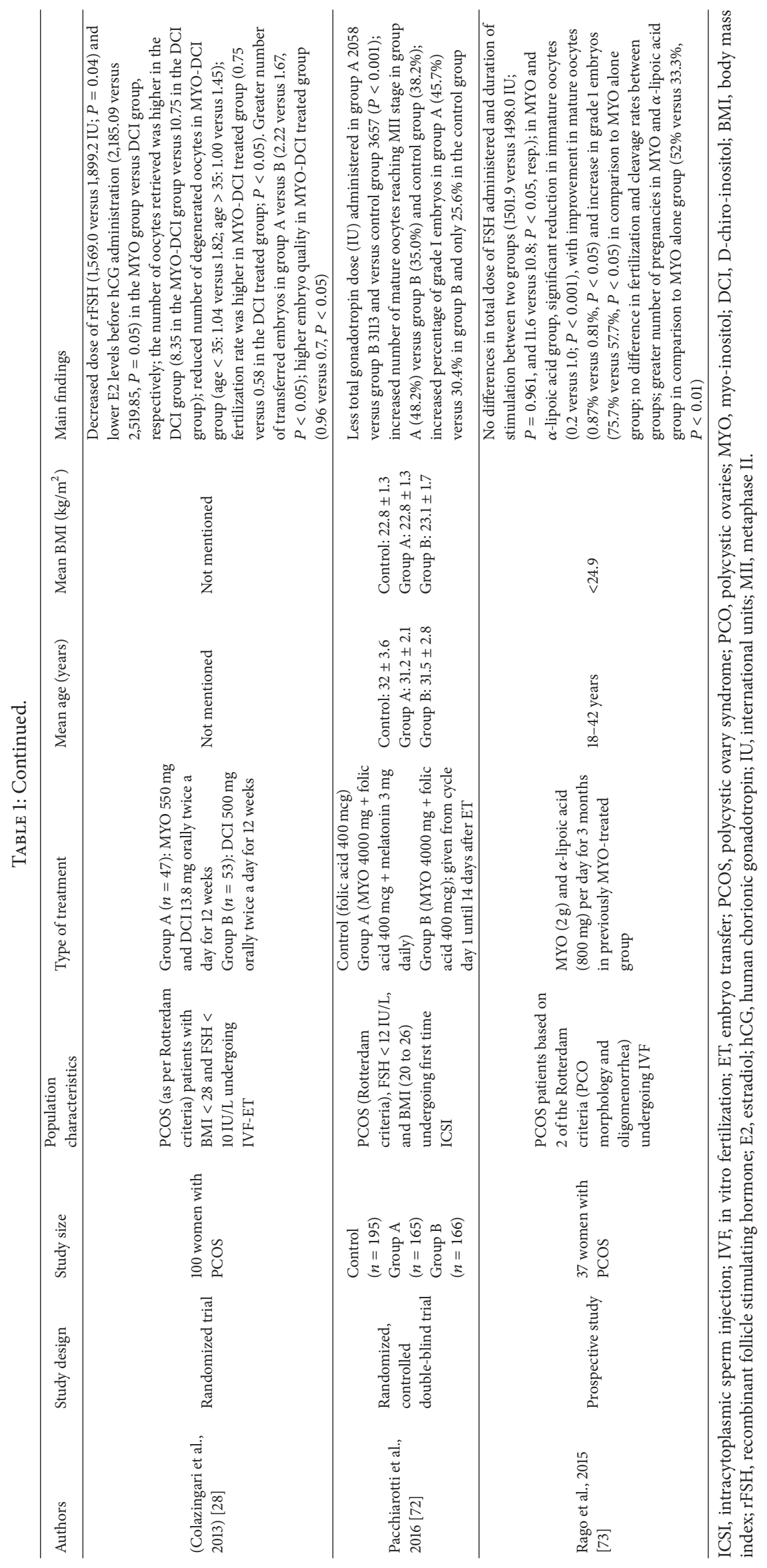


trials on the effects of inositols on ART outcomes in PCOS women.

In summary, current data on inositol supplementation and ART outcomes in women with PCOS is limited to small randomized clinical trials which suggest beneficial effects of MYO on folliculogenesis with improved oocyte maturation and embryo quality. However, data on the effects of MYO supplementation on implantation, pregnancy, and live birth rates following ART in these women is scarce. Thus, large clinical trials aimed at assessing these important ART outcomes are needed. In addition, further studies are needed to determine the optimal ratio of administered MYO : DCI resulting in maximal beneficial effect.

\section{Conclusions}

In conclusion, myo-inositol is an insulin sensitizer which appears to have beneficial effects on ovarian function and response to ART in women with PCOS. It induces nuclear and cytoplasmic oocyte maturation and promotes embryo development. In contrast, D-chiro-inositol appears to exert opposite and detrimental effects on the ovary. While accumulating evidence suggests that myo-inositol improves the number of mature oocytes retrieved, oocyte quality, and embryo quality in women with PCOS undergoing ART, data on its effects on pregnancy and live birth rates in these women is much more limited. Further research on larger patient populations is needed to determine whether inositol supplementation, possibly in combination with other drugs, could improve clinical pregnancy and live birth rates in PCOS women undergoing ART. It is an affordable, widely available, and easy to administer agent which has the potential of improving the outcomes of fertility treatments in women with PCOS.

\section{Competing Interests}

The authors declare that they have no competing interests.

\section{References}

[1] E. S. Knochenhauer, T. J. Key, M. Kahsar-Miller, W. Waggoner, L. R. Boots, and R. Azziz, "Prevalence of the polycystic ovary syndrome in unselected black and white women of the Southeastern United States: A Prospective Study," The Journal of Clinical Endocrinology and Metabolism, vol. 83, no. 9, pp. 30783082, 1998.

[2] B. C. J. M. Fauser, "Revised 2003 consensus on diagnostic criteria and long-term health risks related to polycystic ovary syndrome," Fertility and Sterility, vol. 81, no. 1, pp. 19-25, 2004.

[3] R. Tal, D. B. Seifer, and A. Arici, "The emerging role of angiogenic factor dysregulation in the pathogenesis of polycystic ovarian syndrome," Seminars in Reproductive Medicine, vol. 33, no. 3, pp. 195-207, 2015.

[4] A. Dunaif, K. R. Segal, W. Futterweit, and A. Dobrjansky, "Profound peripheral insulin resistance, independent of obesity, in polycystic ovary syndrome," Diabetes, vol. 38, no. 9, pp. 1165$1174,1989$.
[5] S. Toprak, A. Yönem, B. Çakir et al., "Insulin resistance in nonobese patients with polycystic ovary syndrome," Hormone Research, vol. 55, no. 2, pp. 65-70, 2001.

[6] J. K. Zawadski and A. Dunaif, "Diagnostic criteria for polycystic ovary syndrome: towards a rational approach," in Polycystic Ovary Syndrome, A. Dunaif, J. R. Givens, F. P. Haseltine, and G. R. Merriam, Eds., pp. 377-384, Blackwell Scientific, Boston, Mass, USA, 1992.

[7] M. Ciampelli, A. M. Fulghesu, F. Cucinelli et al., "Impact of insulin and body mass index on metabolic and endocrine variables in polycystic ovary syndrome," Metabolism: Clinical and Experimental, vol. 48, no. 2, pp. 167-172, 1999.

[8] A. D. Genazzani, C. Battaglia, B. Malavasi, C. Strucchi, F. Tortolani, and O. Gamba, "Metformin administration modulates and restores luteinizing hormone spontaneous episodic secretion and ovarian function in nonobese patients with polycystic ovary syndrome," Fertility and Sterility, vol. 81, no. 1, pp. 114-119, 2004.

[9] V. De Leo, A. La Marca, and F. Petraglia, "Insulin-lowering agents in the management of polycystic ovary syndrome," Endocrine Reviews, vol. 24, no. 5, pp. 633-667, 2003.

[10] A. D. Genazzani, C. Lanzoni, F. Ricchieri, and V. M. Jasonni, "Myo-inositol administration positively affects hyperinsulinemia and hormonal parameters in overweight patients with polycystic ovary syndrome," Gynecological Endocrinology, vol. 24, no. 3, pp. 139-144, 2008.

[11] E. Papaleo, V. Unfer, J.-P. Baillargeon, F. Fusi, F. Occhi, and L. De Santis, "Myo-inositol may improve oocyte quality in intracytoplasmic sperm injection cycles. A prospective, controlled, randomized trial," Fertility and Sterility, vol. 91, no. 5, pp. 17501754, 2009.

[12] S. Gerli, E. Papaleo, A. Ferrari, and G. C. di Renzo, "Randomized, double blind placebo-controlled trial: effects of Myoinositol on ovarian function and metabolic factors in women with PCOS," European Review for Medical and Pharmacological Sciences, vol. 11, no. 5, pp. 347-354, 2007.

[13] I. Vucenik and A. M. Shamsuddin, "Protection against cancer by dietary $\mathrm{IP}_{6}$ and inositol," Nutrition and Cancer, vol. 55 , no. 2 , pp. 109-125, 2006.

[14] R. S. Clements Jr. and B. Darnell, "Myo-inositol content of common foods: development of a high-myo-inositol diet," The American Journal of Clinical Nutrition, vol. 33, no. 9, pp. 19541967, 1980.

[15] J. E. F. Reynolds, Martindale: The Extra Pharmacopoeia 30, Rittenhouse Book Distributors, King of Prussia, Pa, USA, 1993.

[16] J. E. Nestler, D. J. Jakubowicz, P. Reamer, R. D. Gunn, and G. Allan, "Ovulatory and metabolic effects of D-chiro-inositol in the polycystic ovary syndrome," The New England Journal of Medicine, vol. 340, no. 17, pp. 1314-1320, 1999.

[17] M. Minozzi, G. D’Andrea, and V. Unfer, “Treatment of hirsutism with myo-inositol: A Prospective Clinical Study," Reproductive BioMedicine Online, vol. 17, no. 4, pp. 579-582, 2008.

[18] E. Papaleo, V. Unfer, J. P. Baillargeon, and T. T. Chiu, "Contribution of myo-inositol to reproduction," European Journal of Obstetrics Gynecology and Reproductive Biology, vol. 147, no. 2, pp. 120-123, 2009.

[19] G. E. Gillaspy, J. S. Keddie, K. Oda, and W. Gruissem, "Plant inositol monophosphatase is a lithium-sensitive enzyme encoded by a multigene family," Plant Cell, vol. 7, no. 12, pp. 2175-2185, 1995.

[20] T.-H. Sun, D. B. Heimark, T. Nguygen, J. L. Nadler, and J. Larner, "Both myo-inositol to chiro-inositol epimerase activities and 
chiro-inositol to myo-inositol ratios are decreased in tissues of GK type 2 diabetic rats compared to Wistar controls," Biochemical and Biophysical Research Communications, vol. 293, no. 3, pp. 1092-1098, 2002.

[21] M. M. Zacchè, L. Caputo, S. Filippis, G. Zacchè, M. Dindelli, and A. Ferrari, "Efficacy of myo-inositol in the treatment of cutaneous disorders in young women with polycystic ovary syndrome," Gynecological Endocrinology, vol. 25, no. 8, pp. 508513, 2009.

[22] D. Costantino, G. Minozzi, F. Minozzi, and C. Guaraldi, "Metabolic and hormonal effects of myo-inositol in women with polycystic ovary syndrome: a double-blind trial," European Review for Medical and Pharmacological Sciences, vol. 13, no. 2, pp. 105-110, 2009.

[23] E. Papaleo, V. Unfer, J.-P. Baillargeon et al., "Myo-inositol in patients with polycystic ovary syndrome: a novel method for ovulation induction," Gynecological Endocrinology, vol. 23, no. 12, pp. 700-703, 2007.

[24] P. T. Goud, A. P. Goud, P. Van Oostveldt, and M. Dhont, "Presence and dynamic redistribution of type I inositol 1,4,5trisphosphate receptors in human oocytes and embryos during in-vitro maturation, fertilization and early cleavage divisions," Molecular Human Reproduction, vol. 5, no. 5, pp. 441-451, 1999.

[25] M. J. Iuorno, D. J. Jakubowicz, J.-P. Baillargeon et al., "Effects of D-chiro-inositol in lean women with the polycystic ovary syndrome," Endocrine Practice, vol. 8, no. 6, pp. 417-423, 2002.

[26] R. Chattopadhayay, A. Ganesh, J. Samanta, S. K. Jana, B. N. Chakravarty, and K. Chaudhury, "Effect of follicular fluid oxidative stress on meiotic spindle formation in infertile women with polycystic ovarian syndrome," Gynecologic and Obstetric Investigation, vol. 69, no. 3, pp. 197-202, 2010.

[27] J.-P. Baillargeon, M. J. Iuorno, D. J. Jakubowicz, T. Apridonidze, N. He, and J. E. Nestler, "Metformin therapy increases insulinstimulated release of D-chiro-inositol-containing inositolphosphoglycan mediator in women with polycystic ovary syndrome," The Journal of Clinical Endocrinology and Metabolism, vol. 89, no. 1, pp. 242-249, 2004.

[28] S. Colazingari, M. Treglia, R. Najjar, and A. Bevilacqua, "The combined therapy myo-inositol plus D-chiro-inositol, rather than D-chiro-inositol, is able to improve IVF outcomes: results from a randomized controlled trial," Archives of Gynecology and Obstetrics, vol. 288, no. 6, pp. 1405-1411, 2013.

[29] A. Bevilacqua and M. Bizzarri, "Physiological role and clinical utility of inositols in polycystic ovary syndrome," Best Practice \& Research. Clinical Obstetrics \& Gynaecology, 2016.

[30] M. J. Coady, B. Wallendorff, D. G. Gagnon, and J.-Y. Lapointe, "Identification of a novel $\mathrm{Na}^{+} /$myo-inositol cotransporter," The Journal of Biological Chemistry, vol. 277, no. 38, pp. 35219-35224, 2002.

[31] R. E. Ostlund Jr., R. Seemayer, S. Gupta, R. Kimmel, E. L. Ostlund, and W. R. Sherman, "A stereospecific myo-inositol/Dchiro-inositol transporter in HepG2 liver cells: identification with D-chiro- $\left[3-{ }^{3} \mathrm{H}\right]$ inositol," The Journal of Biological Chemistry, vol. 271, no. 17, pp. 10073-10078, 1996.

[32] A. Pesty, B. Lefevre, J. Kubiak, G. Geraud, J. Tesarik, and B. Maro, "Mouse oocyte maturation is affected by lithium via the polyphosphoinositide metabolism and the microtubule network," Molecular Reproduction and Development, vol. 38, no. 2, pp. 187-199, 1994.

[33] S. DeLisle, O. Blondel, F. J. Longo, W. E. Schnabel, G. I. Bell, and M. J. Welsh, "Expression of inositol 1,4,5-trisphosphate receptors changes the Ca2+ signal of Xenopus oocytes," The
American Journal of Physiology, vol. 270, no. 4, part 1, pp. C1255C1261, 1996.

[34] M. Herbert, A. P. Murdoch, and J. I. Gillespie, “The thiol reagent, thimerosal induces intracellular calcium oscillations in mature human oocytes," Human Reproduction, vol. 10, no. 8, pp. 21832186, 1995.

[35] M. Sousa, A. Barros, and J. Tesarik, "Developmental changes in calcium dynamics, protein kinase $\mathrm{C}$ distribution and endoplasmic reticulum organization in human preimplantation embryos," Molecular Human Reproduction, vol. 2, no. 12, pp. 967-977, 1996.

[36] T. T. Y. Chiu and P. P. L. Tam, "A correlation of the outcome of clinical in vitro fertilization with the inositol content and embryotrophic properties of human serum," Journal of Assisted Reproduction and Genetics, vol. 9, no. 6, pp. 524-530, 1992.

[37] T. T. Y. Chiu, M. S. Rogers, C. Briton-Jones, and C. Haines, "Effects of myo-inositol on the in-vitro maturation and subsequent development of mouse oocytes," Human Reproduction, vol. 18, no. 2, pp. 408-416, 2003.

[38] S. Colazingari, M. T. Fiorenza, G. Carlomagno, R. Najjar, and A. Bevilacqua, "Improvement of mouse embryo quality by myoinositol supplementation of IVF media," Journal of Assisted Reproduction and Genetics, vol. 31, no. 4, pp. 463-469, 2014.

[39] F. Caprio, M. D. D’Eufemia, C. Trotta et al., "Myo-inositol therapy for poor-responders during IVF: a prospective controlled observational trial," Journal of Ovarian Research, vol. 8, no. 1, article 37, 2015.

[40] M. Minozzi, D. Costantino, C. Guaraldi, and V. Unfer, "The effect of a combination therapy with myo-inositol and a combined oral contraceptive pill versus a combined oral contraceptive pill alone on metabolic, endocrine, and clinical parameters in polycystic ovary syndrome," Gynecological Endocrinology, vol. 27, no. 11, pp. 920-924, 2011.

[41] L. Ciotta, M. Stracquadanio, I. Pagano, A. Carbonaro, M. Palumbo, and F. Gulino, "Effects of Myo-Inositol supplementation on oocyte's quality in PCOS patients: a double blind trial," European Review for Medical and Pharmacological Sciences, vol. 15 , no. 5, pp. 509-514, 2011.

[42] G. A. Burghen, J. R. Givens, and A. E. Kitabchi, "Correlation of hyperandrogenism with hyperinsulinism in polycystic ovarian disease," Journal of Clinical Endocrinology and Metabolism, vol. 50, no. 1, pp. 113-116, 1980.

[43] A. Dunaif, "Insulin resistance and the polycystic ovary syndrome: mechanism and implications for pathogenesis," Endocrine Reviews, vol. 18, no. 6, pp. 774-800, 1997.

[44] A. Dunaif, "Insulin resistance in women with polycystic ovary syndrome," Fertility and Sterility, vol. 86, no. 1, pp. S13-S14, 2006.

[45] M. J. Iuorno and J. E. Nestler, "Insulin-lowering drugs in polycystic ovary syndrome," Obstetrics and Gynecology Clinics of North America, vol. 28, no. 1, pp. 153-164, 2001.

[46] L. C. Huang, M. C. Fonteles, D. B. Houston, C. Zhang, and J. Larner, "Chiroinositol deficiency and insulin resistance. III. Acute glycogenic and hypoglycemic effects of two inositol phosphoglycan insulin mediators in normal and streptozotocindiabetic rats in vivo," Endocrinology, vol. 132, no. 2, pp. 652-657, 1993.

[47] H. K. Ortmeyer, "Dietary myoinositol results in lower urine glucose and in lower postprandial plasma glucose in obese insulin resistant rhesus monkeys," Obesity Research, vol. 4, no. 6, pp. 569-575, 1996. 
[48] J.-P. Baillargeon, E. Diamanti-Kandarakis, R. E. Ostlund Jr., T. Apridonidze, M. J. Iuorno, and J. E. Nestler, "Altered D-chiroinositol urinary clearance in women with polycystic ovary syndrome," Diabetes Care, vol. 29, no. 2, pp. 300-305, 2006.

[49] J.-P. Baillargeon, J. E. Nestler, R. E. Ostlund, T. Apridonidze, and E. Diamanti-Kandarakis, "Greek hyperinsulinemic women, with or without polycystic ovary syndrome, display altered inositols metabolism," Human Reproduction, vol. 23, no. 6, pp. 1439-1446, 2008

[50] J.-P. Baillargeon, M. J. Iuorno, T. Apridonidze, and J. E. Nestler, "Uncoupling between insulin and release of a D-chiro-inositolcontaining inositolphosphoglycan mediator of insulin action in obese women with polycystic ovary syndrome," Metabolic Syndrome and Related Disorders, vol. 8, no. 2, pp. 127-136, 2010.

[51] J. E. Nestler, "Inositolphosphoglycans (IPGs) as mediators of insulin's steroidogenic actions," Journal of Basic and Clinical Physiology and Pharmacology, vol. 9, no. 2-4, pp. 197-204, 1998.

[52] J. E. Nestler, D. J. Jakubowicz, and M. J. Iuorno, "Role of inositolphophoglycan mediators of insulin action in the polycystic ovary syndrome," Journal of Pediatric Endocrinology \& Metabolism, vol. 13, no. 5, pp. 1295-1298, 2000.

[53] K. I. Cheang, J.-P. Baillargeon, P. A. Essah et al., "Insulinstimulated release of d-chiro-inositol-containing inositolphosphoglycan mediator correlates with insulin sensitivity in women with polycystic ovary syndrome," Metabolism: Clinical and Experimental, vol. 57, no. 10, pp. 1390-1397, 2008.

[54] R. Isabella and E. Raffone, "Does ovary need D-chiro-inositol?" Journal of Ovarian Research, vol. 5, article 14, 2012.

[55] Y. Pak, L. C. Huang, K. J. Lilley, and J. Larner, "In Vivo conversion of $[3 \mathrm{H}]$ myoinositol to $[3 \mathrm{H}]$ chiroinositol in rat tissues," The Journal of Biological Chemistry, vol. 267, no. 24, pp. 16904-16910, 1992.

[56] J. Larner, "D-chiro-inositol-its functional role in insulin action and its deficit in insulin resistance," International Journal of Experimental Diabetes Research, vol. 3, no. 1, pp. 47-60, 2002.

[57] J. Larner and J. W. Craig, "Urinary myo-inositol-to-chiroinositol ratios and insulin resistance," Diabetes Care, vol. 19, no. 1, pp. 76-78, 1996.

[58] I. Asplin, G. Galasko, and J. Larner, "chiro-Inositol deficiency and insulin resistance: a comparison of the chiro-inositol- and the myo-inositol-containing insulin mediators isolated from urine, hemodialysate, and muscle of control and type II diabetic subjects," Proceedings of the National Academy of Sciences of the United States of America, vol. 90, no. 13, pp. 5924-5928, 1993.

[59] S. Rice, N. Christoforidis, C. Gadd et al., "Impaired insulindependent glucose metabolism in granulosa-lutein cells from anovulatory women with polycystic ovaries," Human Reproduction, vol. 20, no. 2, pp. 373-381, 2005.

[60] K. Harwood, P. Vuguin, and J. DiMartino-Nardi, "Current approaches to the diagnosis and treatment of polycystic ovarian syndrome in youth," Hormone Research, vol. 68, no. 5, pp. 209217, 2007.

[61] D. Heimark, J. McAllister, and J. Larner, "Decreased myoinositol to chiro-inositol $(\mathrm{M} / \mathrm{C})$ ratios and increased $\mathrm{M} / \mathrm{C}$ epimerase activity in PCOS theca cells demonstrate increased insulin sensitivity compared to controls," Endocrine Journal, vol. 61, no. 2, pp. 111-117, 2014.

[62] S. Dinicola, T. T. Y. Chiu, V. Unfer, G. Carlomagno, and M. Bizzarri, "The rationale of the myo-inositol and D-chiroinositol combined treatment for polycystic ovary syndrome," Journal of Clinical Pharmacology, vol. 54, no. 10, pp. 1079-1092, 2014.
[63] Z. Kamenov, G. Kolarov, A. Gateva, G. Carlomagno, and A. D. Genazzani, "Ovulation induction with myo-inositol alone and in combination with clomiphene citrate in polycystic ovarian syndrome patients with insulin resistance," Gynecological Endocrinology, vol. 31, no. 2, pp. 131-135, 2015.

[64] P. G. Artini, O. M. Di Berardino, F. Papini et al., "Endocrine and clinical effects of myo-inositol administration in polycystic ovary syndrome. A randomized study," Gynecological Endocrinology, vol. 29, no. 4, pp. 375-379, 2013.

[65] M. Minozzi, M. Nordio, and R. Pajalich, "The combined therapy myo-inositol plus D-chiro-inositol, in a physiological ratio, reduces the cardiovascular risk by improving the lipid profile in PCOS patients," European Review for Medical and Pharmacological Sciences, vol. 17, no. 4, pp. 537-540, 2013.

[66] M. Nordio and E. Proietti, "The Combined therapy with myoinositol and D-Chiro-inositol reduces the risk of metabolic disease in PCOS overweight patients compared to myo-inositol supplementation alone," European Review for Medical and Pharmacological Sciences, vol. 16, no. 5, pp. 575-581, 2012.

[67] B. K. Arya, A. U. Haq, and K. Chaudhury, "Oocyte quality reflected by follicular fluid analysis in poly cystic ovary syndrome (PCOS): a hypothesis based on intermediates of energy metabolism," Medical Hypotheses, vol. 78, no. 4, pp. 475-478, 2012.

[68] F. Ferrari, F. Facchinetti, A. E. Ontiveros et al., "The effect of combined inositol supplementation on maternal metabolic profile in pregnancies complicated by metabolic syndrome and obesity," American Journal of Obstetrics and Gynecology, 2016.

[69] M. Bizzarri and G. Carlomagno, "Inositol: history of an effective therapy for polycystic ovary syndrome," European Review for Medical and Pharmacological Sciences, vol. 18, no. 13, pp. 18961903, 2014.

[70] V. Unfer, G. Carlomagno, P. Rizzo, E. Raffone, and S. Roseff, "Myo-inositol rather than D-chiro-inositol is able to improve oocyte quality in intracytoplasmic sperm injection cycles. A prospective, controlled, randomized trial," European Review for Medical and Pharmacological Sciences, vol. 15, no. 4, pp. 452-457, 2011.

[71] G. Carlomagno, V. Unfer, and S. Roseff, "The D-chiro-inositol paradox in the ovary," Fertility and Sterility, vol. 95, no. 8, pp. 2515-2516, 2011.

[72] A. Pacchiarotti, G. Carlomagno, G. Antonini, and A. Pacchiarotti, "Effect of myo-inositol and melatonin versus myoinositol, in a randomized controlled trial, for improving in vitro fertilization of patients with polycystic ovarian syndrome," Gynecological Endocrinology, vol. 32, no. 1, pp. 69-73, 2016.

[73] R. Rago, I. Marcucci, G. Leto et al., "Effect of myo-inositol and alpha-lipoic acid on oocyte quality in polycystic ovary syndrome non-obese women undergoing in vitro fertilization: a pilot study," Journal of Biological Regulators and Homeostatic Agents, vol. 29, no. 4, pp. 913-923, 2015. 


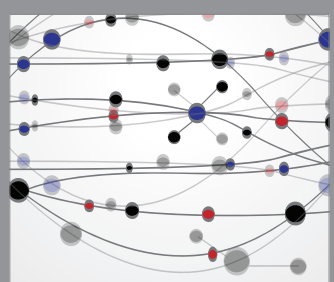

The Scientific World Journal
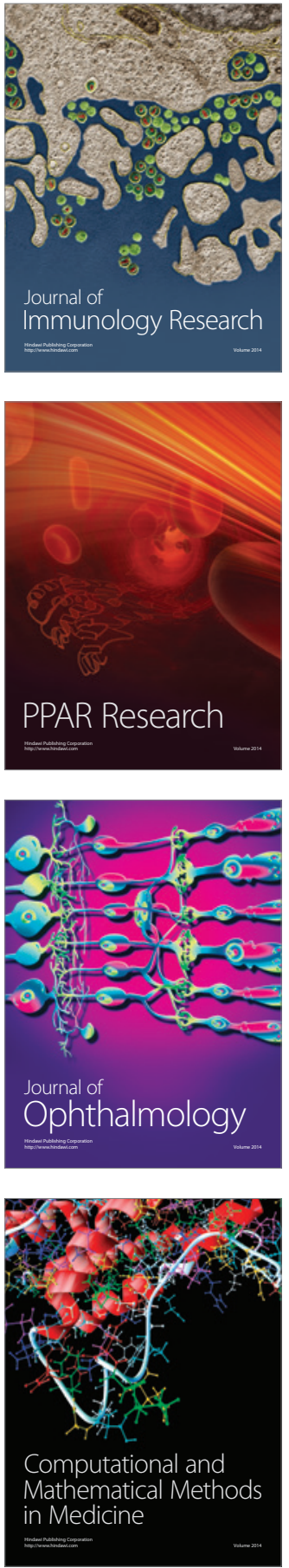

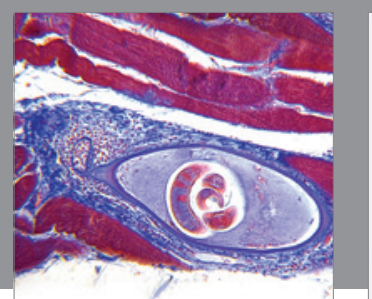

Gastroenterology Research and Practice

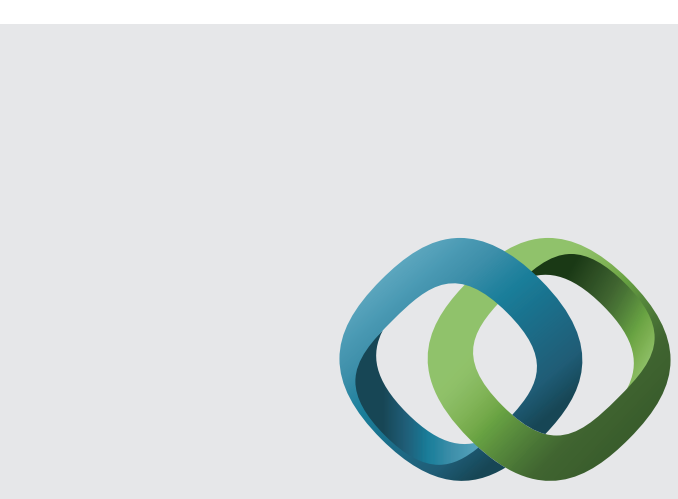

\section{Hindawi}

Submit your manuscripts at

http://www.hindawi.com
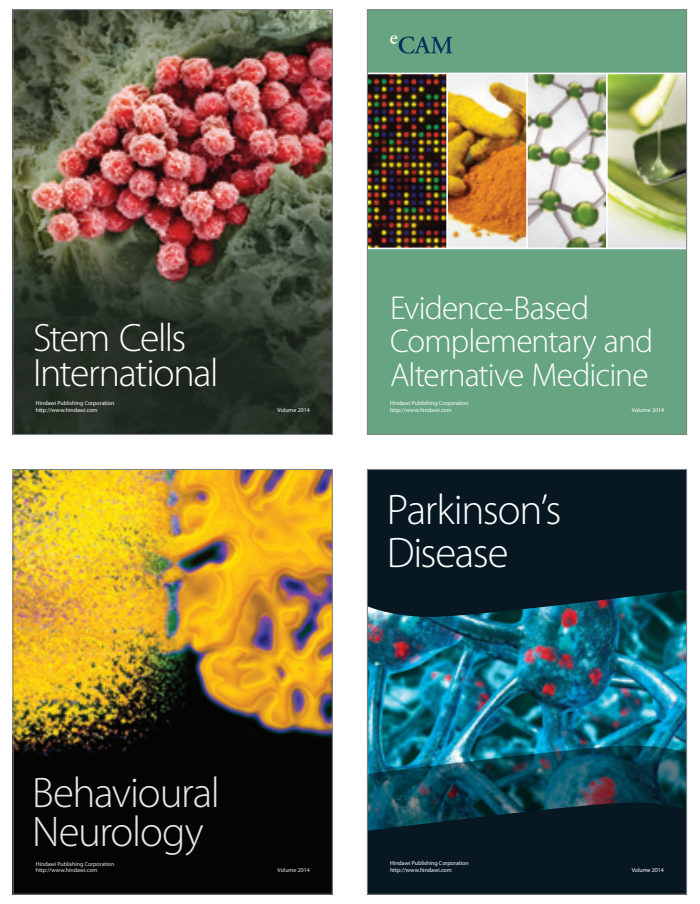
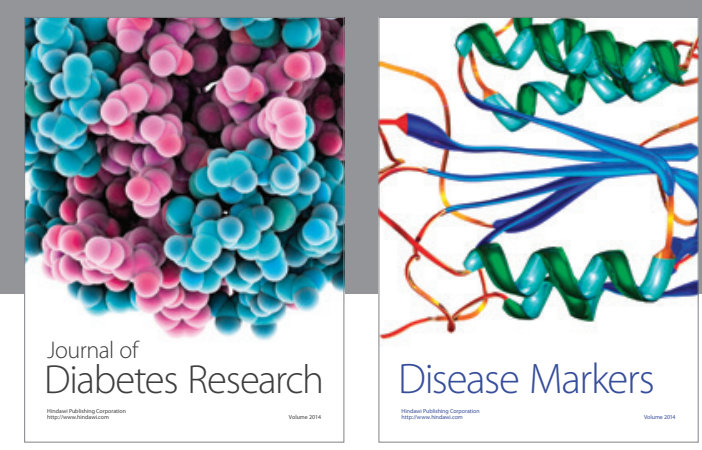

Disease Markers
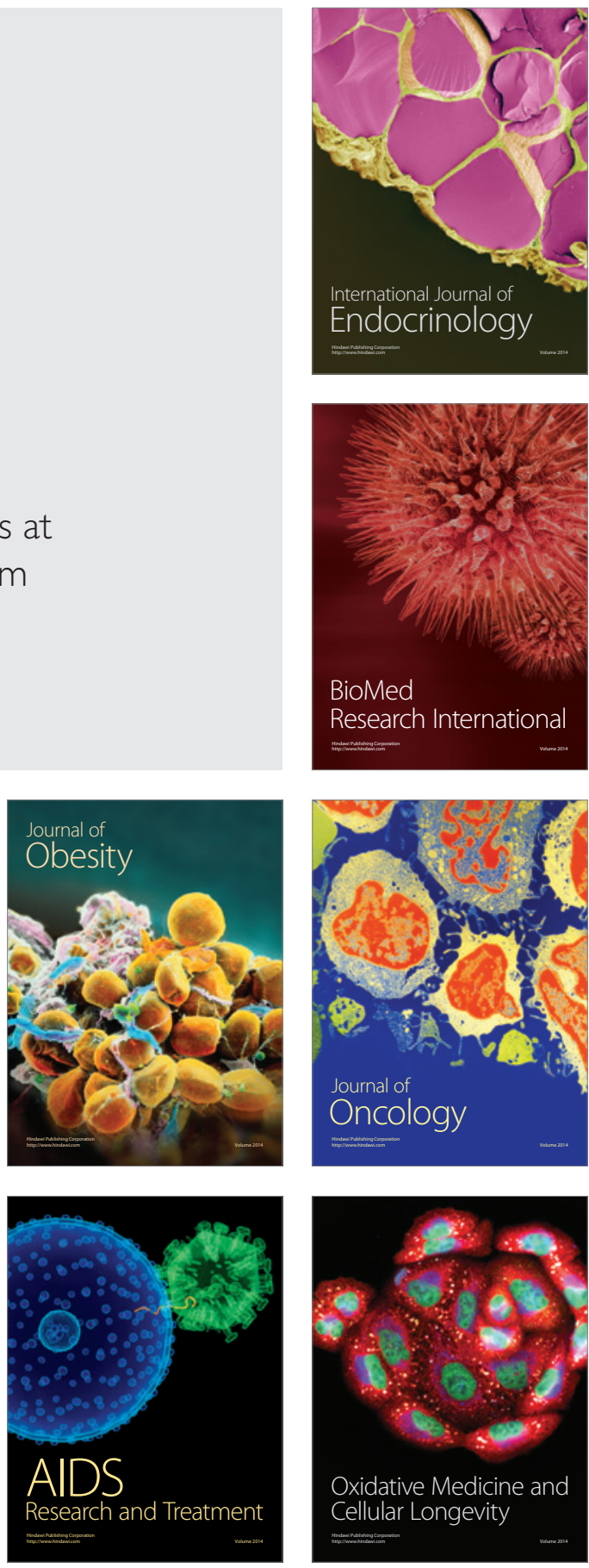